\title{
Kurt Singer - Karl Wolfskehl - „Der Mensch ist ein Ganzes, eins in jeder seiner unterschiedlichen Erscheinungen, eins auf jedem seiner unterschiedlichen Wege ${ }^{\star 1}$
}

Das Ziel der folgenden Überlegungen ist zum einen, die Persönlichkeit und - kursorisch - das Werk Kurt Singers vorzustellen, als jemand, der das Schicksal Karl Wolfskehls teilte und sich zudem eini-ge Zeit in seiner geographischen Nähe befand. Zum anderen soll konkret die Beziehung zwischen Kurt Singer und Karl Wolfskehl dargelegt werden. Beide gehören in den Umkreis Stefan Georges und stehen sich eine Zeitlang zumindest brieflich nahe.

Dabei wird im folgenden, diese Analyse nicht von der literaturwissenschaftlichen Seite her vorgenommen. Vielmehr steht ein japanologischer Ansatz im Vordergrund. Auf den ersten Blick erscheint dies vielleicht etwas fragwürdig: Was hat Karl Wolfskehl mit Japan zu tun? Über die Persönlichkeit Kurt Singers gibt es durchaus Berührungspunkte mit Japan, wie im folgenden im einzelnen dargelegt wird.

Da die Popularität Kurt Singers als Wissenschaftler und poetisch Tätiger kaum ein breites Püblikum erreicht hat, wird zunächst der biographische Werdegang Singers kurz skizziert. Innerhalb dieses biographischen Rasters lassen sich $u$. a. seine Werke in einen Kontext einordnen, und auf diese Weise werden Hintergründe für persönliche Kontakte wie denjenigen zu Karl Wolfskehl deutlich.

Wie noch zu zeigen sein wird, ist Singers Schaffen außerordentlich vielschichtig. Von Hause aus Nationalökonom publizierte er nicht nur $\mathrm{zu}$ wirtschaftswissenschaftlichen Themen, sondern auch Betrachtungen zum klassischen Altertum, anthropologische Arbeiten besonders über Japan - und Überlegungen zu jüdischem Kulturgut. Im vorliegenden Rahmen kann und soll keine umfassende Betrachtung seines Werkes vorgenommen werden. Vielmehr werden einige seiner japanbezogenen Arbeiten in den Mittelpunkt gestellt, da diese besonders in der Beziehung zu Wolfskehl eine Rolle spielen. Methodisch erscheint es zudem sinnvoll, von der überblicksartigen Behandlung von Singers Schaffen abzugehen. Es haben sich dabei

1 Singer, Kurt: Spiegel, Schwert und Edelstein. Strukturen des japanischen Lebens. Hg., aus dem Englischen übersetzt und mit einer Einführung versehen von Wolfgang Wilhelm, Frankfurt a. M. 1991, S.69. 
in der Sekundärliteratur bestimmte Betrachtungsweisen eingebürgert, die immer wieder aufgegriffen werden. An dieser Stelle bietet sich die Gelegenheit, solche Stereotype zumindest einmal punktuell zu hinterfragen.

\section{Zu Singers Biographie:}

Kurt Singer wurde am 18. 5. 1886 als Sohn des jüdischen Ehepaares Maximilian Singer und Mary, geb. Peartree, deren Eltern nach England ausgewandert waren, in Magdeburg geboren. Er hatte eine jüngere Schwester, Edith, die, laut einer biographischen Notiz Peter Landmanns in Basel, 1941 aus Hamburg verschleppt und wahrscheinlich im Konzentrationslager Lietzmannstadt ermordet wurde. Sein Vater war 1878 als Kompagnon in eine Lederhandlung eingetreten und war 1910 offensichtlich Inhaber eines Bekleidungsgeschäftes. ${ }^{2}$

Kurt Singer legte 1904 seine Reifeprüfung in Magdeburg ab. Danach studierte er bis 1910 an den Universitäten in Berlin, Freiburg, Straßburg und Genf. Unter den akademischen Lehrern, die einen großen Einfluß auf ihn hatten, waren Georg Friedrich Knapp, Georg Simmel und Heinrich Wölfflin. Die Fächer, die ihn besonders faszinierten, waren Nationalökonomie, aber auch Soziologie, Literaturwissenschaft, Kunstgeschichte und Philosophie. 1910 wurde er in Straßburg mit einer Arbeit über die indische Geldreform bei Georg Friedrich Knapp zum Dr. rer. pol. promoviert.

Von 1910 bis 1912 arbeitete er als wissenschaftlicher Sekretär am amerikanischen Konsulat in Chemnitz. Ab 1912 wurde er Assistent von Friedrich Bendixen, des Direktors der Hamburger Hypothekenbank. In dieser Zeit nahm er einige zusätzliche Aufgaben an. 1913 wurde er Chefredakteur des Handels- und Finanzteils des Hamburgischen Correspondenten. Dies sah er als willkommene Gelegenheit, die Geldschöpfungslehre Knapps zu verfechten, was ihm in akademischen Kreisen den Ruf des Häretikers eintrug. Aber der Erste Weltkrieg bestätigte seine Zweifel an der vorherrschenden Wirtschaftstheorie. 1917 trat Singer in die Redaktion der Zeitschrift Wirtschaftsdienst ein. ${ }^{3}$ Diese war von dem Bankier Max Warburg gegründet worden, dessen Bruder Aby Warburg ein späterer Kollege Singers an der Hamburger Universität war. Herausgegeben wur-

2 Eschbach, Achim: "Kurt Singer und die moderne Semiotik", unveröffentlịchtes Typoskript im Stuttgarter George-Archiv, 14 S., StGA, o. J., S. 1.

3 Ôta, Hidemichi: "Kurto Jingâ no shôgai to gakumon (Kurt Singers Leben und Studien)", in: Tôkyo University Press 149 (1985), S. 18 f. 
de der Wirtschaftsdienst vom Hamburgischen Welt-Wirtschafts-Archiv, das damals noch Zentralstelle des Hamburgischen Kolonialinstituts hieß. Die Funktion dieses Organs war der des Londoner Economists vergleichbar. Es sollte - an eine gebildete Leserschaft gerichtet - zwischen ökonomischer Theorie und Praxis vermittelt werden. Singer war zunächst Mitarbeiter in der Redaktion und wurde 1919 einer der beiden Hauptschriftleiter. Diese Aufgabe erfüllte er bis 1928. In dieser Funktion kam Singer in Kontakt mit John Maynard Keynes in Cambridge. Singer korrespondierte mit ihm, übersetzte einige von Keynes Artikeln für den Wirtschaftsdienst, und es ist nicht unwahrscheinlich, daß Singers spätere Entscheidung, nach Australien zu gehen, mit von Keynes beeinflußt war. ${ }^{4}$ Zunächst habilitierte Kurt Singer sich 1920 in Hamburg mit einer Arbeit über Das Geld als Zeichen, die im gleichen Jahr bei Fischer in Jena publiziert wurde. Danach hielt Singer an der Hamburger Universität Vorlesungen über Bank- und Börsenwesen sowie soziologische Themen. 1924 wurde der Privatdozent Kurt Singer zum außerordentlichen Professor ernannt. Ein Jahr später erhielt er einen Ruf an die Handelshochschule in Königsberg, den er jedoch ablehnte. Einerseits hatte ihm Knapp davon abgeraten, nach Königsberg zu gehen, mit der Begründung, Singers Wirksamkeit sei in Hamburg größer. Andererseits arbeitete Singer zu diesem Zeitpunkt an seiner Studie Platon der Gründer. ${ }^{5}$ Ganz aus Georgischer Perspektive geschrieben, war diese Arbeit für Singer selbst ungeheuer wichtig, und er fürchtete Verzögerungen durch einen Ortsund Arbeitswechsel. Dieses Buch erschien 1927.

An dieser Stelle soll nur eine kurze Anmerkung zu Singers Verhältnis zu George gemacht werden. In der Sekundärliteratur existieren unterschiedliche Ansichten über den Kontakt zwischen beiden. Nach Wolfgang Wilhelm 6 macht Singers Studienfreund Ernst Robert Curtius ihn in Straßburg mit den Blättern für die Kunst vertraut und führt ihn ebenfalls in den Kreis um Stefan George ein. Groppe stellt heraus, daß - laut Anmerkungen des Herausgebers des George-Gundolf-Briefwechsels zu einem Brief Friedrich Gundolfs vom 30. 12. 1916 - Singer durch seine Besprechung von Gundolfs Shakespeare und der deutsche Geist zuerst mit Gundolf und über diesen mit George bekannt wurde.7 Eschbach sichtet Singers Kor-

4 Eschbach, "Kurt Singer", S. 2.

5 Groppe, Carola, Die Macht der Bildung. Das deutsche Bürgertum und der George-Kreis 1890-1933, Köln-Weimar-Wien 1997, S. 518 f.

6 Wilhelm, "Vorwort", S. 9.

7 Groppe, Macht der Bildung, S. 517, Anm. 90. 
respondenz im Stuttgarter George Archiv, dem Deutschen Literaturarchiv in Marbach und an der Hamburger Universität und stellt fest, daß Singer spätestens seit 1912 auf vielfältige Weise mit dem George-Kreis liiert gewesen sein mußte. ${ }^{8}$ Fest steht, daß Singer zum Zeitpunkt der Entstehung von Platon der Gründer stark unter dem Einfluß Georges stand.

In dieser Zeit - darauf weist Eschbach hin ${ }^{9}$ - erhielt dieser ebenfalls den Walther-Rathenau-Preis. Ein genaues Datum ist hier noch nicht festzumachen. ${ }^{10}$

1928 dann schied Singer aus der Redaktion des Wirtschaftsdienstes aus. Inzwischen war das Kieler Institut für Weltwirtschaft Mitherausgeber geworden und Singers kompromißlose Haltung zu inhaltlichen Aspekten hatte zu Problemen mit dessen Leiter, Bernhard Harms, geführt. Harms hatte offensichtlich auch dazu beigetragen, daß Singer den Ruf aus Königsberg erhalten hatte und war über dessen Ablehnung verärgert. Dazu kam, daß Singer eine Abhandlung eines Harms' Mitarbeiters im Wirtschaftsdienst sehr kritisch besprochen hatte. 11

Große Bedeutung für Singers weiteres Geschick hatte eine Einladung der Tokyo Teikoku Daigaku, der kaiserlichen Universität in Tokyo (heute Tokyo Daigaku), die er 1930 erhielt. Am Keizai Gakubu, der wirtschaftswissenschaftlichen Fakultät, sollte er dort vier Jahre lang als visiting professor einen Lehrstuhl einnehmen, der traditionell immer von ausländischen Wissenschaftlern vertreten wurde. 12

Singer nahm diese Einladung an, ließ sich von der Hamburger Universität beurlauben und traf am 20. 5. 1931 in Japan ein. In Tokyo erwarteten ihn ein für damalige japanische Verhältnisse überdurchschnittlich gutes Gehalt mit Wohnkostenzuschuß und Reisegeld. Singer entschied sich kurz nach seiner Ankunft dafür, nicht in Tokyo selbst, sondern im etwa zwei Stunden entfernten Kamakura zu wohnen. Er fand ein traditionelles Haus am Meer und lebte dort während seiner Tokyoter Zeit zurückgezogen mit einer japanischen Haushälterin. Er hatte etwa sechs Stunden Lehrverpflichtung in der Woche zu erfüllen. Seine zentralen Themen waren die National-

8 Eschbach, "Kurt Singer", S. 5.

9 Ebd., S. 2.

10 Vgl. Groppe, Macht der Bildung, S. 518.

11 Pawlowsky, Peter, "Vorwort" zu: Kurt Singer, The Idea of Conflict, vermehrt um Ausgewählte Schriften zu Wirtschaft und Staat, hg. und eingeleitet von Peter Pawlowsky, Tübingen 1973; S. X.

12 Ôta, "Kurto Jinga", in: Tôkyo University Press 145 (1984), S. 20. 
ökonomie Knapps und die soziologischen Theorien Max Webers. Unterrichtssprachen waren Englisch und Deutsch. ${ }^{13}$ Seiner eigenen Einschätzung nach begegneten ihm seine Studenten mit Achtung und Zuneigung, und es gelang ihm, ihnen ,die geometrisch richtige Darstellung der Konsumentenrente, Elastizitäten usw. und das Hantieren mit soziologischen Definitionen Max Webers" beizubringen (Brief Kurt Singers an Eduard Rosenbaum, 4. 3. 1934)14. Schaut man sich die Perspektive seiner damaligen Studenten an, ergibt sich ein etwas abweichender Eindruck. Ôtsuka Hisao z.B. besuchte Singers Veranstaltung zu Max Weber. Er berichtet, daß Singer auf Deutsch unterrichtete und ausschließlich Originaltexte verwendete. Nach den Einführungsstunden blieben alle Studenten bis auf ihn weg. Als er gegen Ende der Veranstaltung erkrankte, wurde diese ganz abgesetzt. ${ }^{15}$ Singers Unterricht war für seine Studenten ganz einfach zu schwierig.

Unterdessen wurde Singer 1933 in Hamburg als Juden auf der Grundlage des rassischen „Gesetzes zur Wiederherstellung des deutschen Berufsbeamtentums“ die venia legendi entzogen. Unter dem Druck des Achsenpartners verlängerte auch die Tokyo Teikoku Daigaku im Jahre 1935 Singers Vertrag nicht noch einmal. Dieser war in der Folge ein Jahr arbeitslos, in dem er u. a. eine dreimonatige Chinareise unternahm. Singer war in dieser Zeit schokkiert über die allgemeine politische Situation in Japan. 16

1936 bekam er eine Stelle als Deutschlehrer an der Dai Ni Koto Gakko in Sendai. Vermutlich war Sendai abgelegen genug, sodaß man ihn hier beschäftigen konnte, ohne daß es offiziell weiter auffiel. Auch aus dieser Zeit existieren Eindrücke seiner ehemaligen Schüler. Ôta berichtet, 17 Singer sei ernst und unnahbar gewesen, sein Unterricht sei äußerst anspruchsvoll gewesen und hätte man ihn auf der Straße getroffen, hätte er statt eines Grußes oft ohne jeden Zusammenhang intellektuelle Fragen gestellt.

In Sendai nahm Singer Kontakt zu Karl Löwith auf, der als jüdischer Philosoph von Marburg über das italienische Exil nach Japan gekommen war und dort an der Tôhoku Daigaku lehrte. Mit ihm

13 Ôta, "Kurto Jinga", in: Tôkyo University Press 145 (1984), S. 21.

14 Vgl. Pawlowsky, "Vorwort", S. XI.

15 Ôta, "Kurto Jinga", in: Tôkyo University Press 145 (1984), S. 22.

16 Singer, Kurt, "Bericht über die japanischen Jahre (1931-1939)", in: Erich Boehringer/Wilhelm Hoffmann (Hg.), Robert Boehringer. Eine Freundesgabe, Tübingen 1957, S. 602 f. und Ôta, "Kurto Jinga", in: Tôkyo University Press 145 (1984), S. 20.

17 Ôta, "Kurto Jinga”, in: Tôkyo University Press 149 (1985), S. 16. 
und anderen Universitätslehrern stand Singer in einem produktiven Austausch, der u. a. in der Anthologie The Life of Ancient Japan, einem Quellenband, der parallel zu seiner Arbeit an Mirror, Sword, and Jewel entstand, resultierte.18 Dieser erschien 1939 beim renommierten Verlag Iwanamishoten. Da Singer lediglich an einer höheren Schule und nicht an der Universität unterrichtete, war seine berufliche Position hier wenig einflußreich. Bis 1939 blieb Singer in Sendai. Obwohl sich Japan in einigen Fällen über rassische Forderungen aus Deutschland hinwegsetzte, war dies bei Singer nicht der Fall. Er wurde 1939 unter dem Vorwurf der „mangelnden pädagogischen Eignung" entlassen. Hier könnte die Tatsache mit hineinspielen, daß Singer offensichtlich Schwierigkeiten innerhalb des japanischen Erziehungssystems hatte. 19

Singer mußte schließlich Japan verlassen. Auswanderungsalternativen waren England, wobei Keynes ihm heftig davon abriet dorthin zu kommen, Amerika, Australien - schließlich befand sich hier Karl Wolfskehl in der Nähe -, und Jerusalem, wo sich Martin Buber aufhielt. Allerdings zerschlug sich hier Singers Hoffnung auf einen Lehrstuhl. Letztendlich entschied er sich für Australien. ${ }^{20}$

Nach 99 Monaten verließ Singer Japan in Richtung auf Canberra. Angekommen in Australien wurde er zunächst als Angehöriger einer feindlichen Macht von Juni 1940 bis Oktober 1941 interniert. Danach ließ Singer sich in Melbourne nieder. Die Dyason Foundation for the Study of Conflict hatte ihm ein Stipendium für seine Studie The Idea of Conflict gegeben, die er Martin Buber widmete und die 1949 in Melbourne erschien.21

Nach dem Krieg 1946 stellte Australien ihn vor die Alternative, entweder die britische Staatsangehörigkeit anzunehmen oder Australien zu verlassen. Für Singer, der sich sicherlich immer zuerst als Deutscher und dann als Jude gesehen hatte, war es eine schwierige Entscheidung. Aber er wurde Brite und konnte von da ab ohne Probleme in Australien leben und arbeiten. 22

Im Juni des gleichen Jahres erhält Singer eine Position als Teaching Fellow am Department of Economics an der University of Sydney. Drei Jahre später, im Juni 1949, wird er hier Temporary Lecturer. 1951 wird er Acting Lecturer und auf dieser Ebene bleibt er bis zu seiner Pensionierung im Jahre 1955.23 Es bleibt festzuhalten, daß er

18 Wilhelm, "Vorwort", S. 16.

19 Ôta, "Kurto Jinga", in: Tôkyo University Press 144 (1984), S. 28.

20 Ôta, "Kurto Jinga", in: Tôkyo University Press 149 (1985), S. 20

21 Wilhelm, "Vorwort", S. 19.

22 Ebd., S. 20. 
nicht - wie es sich in die Sekundärliteratur eingeschlichen hat 24 -, die Universität gewechselt hat und später an der University of New South Wales tätig war. Anläßlich seiner Pensionierung findet sich ein Vermerk im Protokoll eines faculty meetings vom 10. 11. 1955: „It was agreed that the Faculty should place on record its appreciation of the services given by Dr. Singer and an expression of its good wishes to him in his future activities." Bis 1952 war er nicht im Vorlesungsverzeichnis erwähnt, später ist dort seine Qualifikation mit Dr. rer. pol. (Strasbourg, Economics) angegeben. ${ }^{25}$ (lt. Personalakte Kurt Singer, University of Sydney).

$\mathrm{Zu}$ dieser Zeit befanden sich Hans Brasch in Melbourne und Karl Wolfskehl in Auckland, ansonsten ist Singer aber wohl recht einsam gewesen und in Australien immer fremd geblieben. Auch konnte er sich nie an das australische examen-orientierte Fakultätsdasein gewöhnen (Brief an Karl Löwith, 9. 2. 1946). 26

1957 erhielt Singer vom Hamburger Senat eine finanzielle Wiedergutmachung in Form der Bezüge eines emeritierten Professors. Dies gibt Singer die Möglichkeit, Australien zu verlassen und nach Europa zurückzukehren. Nach einem kurzen Aufenthalt in Deutschland reist Singer über die Schweiz und Italien nach Griechenland. Er verlebt einige wenige ruhige, mit Forschung angefüllte Jahre bis er am 14. 2. $1962 \mathrm{im}$ Alter von 75 Jahren in Athen stirbt. 27

Interessant für die vorliegende Fragestellung sind besonders Singers japanische und australische Exilzeit, denn in diese Zeit fällt schwerpunktmäßig der Kontakt mit Karl Wolfskehl. Dies ist zudem genau der Bereich, der in der Singer-Forschung bisher immer eher in den Hintergrund gerückt worden ist. So werden z. B. die persönlichen Erinnerungen seiner japanischen Studenten und Schüler in der westlichen Forschung kaum zur Kenntnis genommen, da sie nur auf Japanisch publiziert sind.

Es steht fest, daß Kurt Singer und Karl Wolfskehl noch in Deutschland zumindest brieflich in Kontakt gestanden haben, darüber liegen d. Verf. allerdings keine Dokumente vor. Karl Wolfskehl

23 Lt. Personalakte Kurt Singer, University of Sydney.

24 Vgl. etwa Wilhelm, "Vorwort", S. 20; Groppe, Die Macht der Bildung, S. 518; Eschbach, "Kurt Singer", S. 3.

25 Ebd.

$26 \mathrm{Vgl}$. Wilhelm, "Vorwort", S. 20.

27 Ôta, Hidemichi, "Atene no Jingâ (Singer in Athen)", in: Tôkyo University Press 160 (1986), S. 15. 
schreibt jedoch am 15. 5. 1939 als Reaktion auf ein Päckchen, das Singer ihm aus Japan geschickt hatte, an Singer: ,... das war eine freudige und wie sinnvolle Überraschung, mit der ich nach Jahren wieder einmal, erblindenden Auges, über die zarten und doch so festen Schriftzüge hintastete und danach begierig den Inhalt des Päckleins überschlug!" $28 \mathrm{Zu}$ diesem Zeitpunkt, als Singer sich offensichtlich nach langer Zeit wieder an Wolfskehl wendete, war Wolfskehl etwa seit einem Jahr in Auckland. Kurt Singers Zeit in Japan näherte sich ihrem Ende. Er war von der Dai Ni Koto Gakko in Sendai entlassen worden und nach Tokyo zurückgekehrt, wo er die ausgeprägt nationalistische Stimmung in Japan beschreibend, „eine Luft“ ${ }^{\star}$ vorfand, ,in der ein Europäer nicht mehr atmen konnte. Das einst so gastliche Land war nun den bösen Geistern erztauben Wahns, urblinden Mißtrauens ausgeliefert. Man schien die bloße Anwesenheit von Fremden als Befleckung des Bodens in der näherrückenden Stunde von Sieg oder Untergang zu empfinden, unfähig, zwischen wahrem Freund und Feind zu unterscheiden". 29

Beim Inhalt des oben erwähnten Päckchens handelte es sich um einen kulturanthropologischen Essay Singers mit dem Titel "Differences between Chinese and Japanese Culture". ${ }^{30}$ Der Form nach eine wissenschaftliche Abhandlung, die jedoch eine Menge poetischer Einsprengsel enthält, stellt Singer hier - erklärend anhand von zahlreichen Beispielen aus Literatur, Architektur, Kalligraphie etc: - seine Ansicht vor, daß die chinesische Kultur die japanische beeinflußt, diese jedoch nicht geprägt habe, denn Japan habe sämtliche Einflüsse adaptiert und assimiliert. Diese Beobachtung hat sicherlich eine gewisșe Gültigkeit, auffallend ist jedoch die Vorgehensweise der Beweisführung Singers. Stark beeinflußt von seiner Grundüberzeugung, daß die Menschheit sich seit alters her bestimmten Präsuppositionen nicht entziehen kann, geht er auch hier von der Prämisse aus, daß traditionelle Künste „truly reveal a nation's temper".31 Dieser Terminus ,a nation's temper" wird weder analysiert noch differenziert. Es stellt sich die Frage nach dem Begriff der Nation und nach historischen, sozialen, geographischen sowie zeitlichen Paradigmen. Es ist hier die Rede von chinesischen „Einflüssen“, „Modellen“, chinesischer Geisteshaltung,32 vom japanischen "Leben" und nirgendwo wird der Tat-

$28 \mathrm{BaN}$ II, S. 325.

29 Singer, "Bericht", S. 603.

30 In: The Yale Review, New Series, Vol. XXVII, 1938, pp. 772-789.

31 Ebd., S. 773. 
sache Rechnung getragen, daß es sich beim chinesischen Reich um ein außerordentlich ausgedehntes, kulturell verschiedenes und historisch äußerst wandelbares Phänomen handelt. Ebenso ist Japan kein monolithischer Block. Diese Sehweise wird später in Mirror, Sword, and Jewel noch weitergetrieben, wenn Singer den ,typischen" Chinesen und den "typischen" Japaner kontrastiert. ${ }^{33}$

An dieser Stelle stellt sich die Frage, wie jemand, der Deutschland verlassen mußte, weil dort genau mit diesen Denkmustern gearbeitet wurde, zwar - zugegebenerweise unter umgekehrtem Vorzeichen -, aber genau in dieser Richtung weiterargumentieren kann. Dieser Essay arbeitet mit Stereotypen, er geht von rassischen Gesichtspunkten aus und er entwickelt das Bild eines Nationalcharakters, der Japan - nach Singer - einzigartig erscheinen läßt: „Many nations have wandered and warred and received floods of foreign immigrants' and warriors' blood: every soil has nurtured in the course of history more than one race of men. But it is only in those rare moments when a creative race united in a common creed, by decree of fate or act of grace, finds a fertile soil, congenial to god and man, that a new archetype of culture arises".34 Auf diesen Aspekt wird später noch einmal eingegangen, zunächst wird Wolfskehls Reaktion auf diesen Essay kurz angesprochen. Wolfskehl bedankt sich in seinem Brief an Singer vom 15. 5. 1939 für die „Erhellung östlicher Geistesart" durch Singers Essay 35 und konstatiert seinen ersten Eindruck folgendermaßen: Singer gehe „behutsam und sicher ins Wesen ein" und Singers "große Kunst, das fast Unsagbare dennoch ins Wort zu bringen", bewähre sich wie nur je. ${ }^{36}$ Hier findet sich kein Wort der Kritik, nicht einmal ein Hinweis im oben angeführten Sinn von jemand, der ebenso intellektuell vorgebildet ist wie Singer, der ebenso unter Deutschland gelitten hat und leidet wie Singer und der gerade auch als Immigrant für diese Dinge ebenso sensibilisiert gewesen sein mußte. Statt dessen ist hier ein Rückzug auf Formeln zu beobachten, es wird in diesem Brief auf antike Bilder rekurriert, und Wolfskehl gibt seiner Bewunderung für Singers hervorragendes Englisch Ausdruck: „Ihr Englisch aber bewundre ich parte di tutt'altro aus der Maßen“. ${ }^{37}$ Er legt außerdem seinem Brief an Singer Gedichte als Gegenge-

32 Ebd.

33 Singer, Spiegel, Schwert und Edelstein, S. $254 \mathrm{f}$.

34 Singer, "Differences", S. 789.

35 BaN II, S. 325.

36 Ebd.

37 Ebd., S. 326. 
schenk bei. Ist diese unkritische Haltung begründet in der Loyalität zweier George-Kreis-Anhänger, die sich - aus den gleichen Gründen aus Deutschland vertrieben - beide unendlich einsam und intellektuell unterfordert fernab der Heimat wiederfinden? Oder ist hier - unabhängig vom aktuellen Adressaten - ein gewisser Rückzug der Persönlichkeit Wolfskehls als Reaktion auf seine Situation zu beobachten, wie dies von Blasberg 38 angedeutet wird? Sind Formeln, Komplimente 39 und Stilisierungen zu deuten als „Masken, hinter denen das Schweigen verborgen ist"? Diese Frage kann im Rahmen der vorliegenden Ausführungen nur angedeutet werden.

Für Singer jedenfalls bedeutete der briefliche Kontakt mit Wolfskehl viel. In seinem „Bericht über die japanischen Jahre“ bemerkt er, $40 \mathrm{da}$ Mitte bis Ende der dreißiger Jahre viele seiner Briefpartner verstummt seien. „Dafür kamen zurufend und wachhaltend, anklagend und berichtend, zärtlich und schwingend, Briefe von Edith Landmann und Karl Wolfskehl, die mir bisher ferner gestanden hatten. Wir fühlten uns keineswegs als vom Göttertisch vertriebne Tantaliden, wie man wohl gewähnt hat ... eher als Athener, die dem eingedrungenen Feind die heilige Stadt überließen, um an schwankem Schiffbord auf ungewissem Element den echten Kampf mit reinen Waffen fortzukämpfen in uns selber nach unsrem Teil die Stadt tragend, einzig im Wort des Dichters Boden findend und Luft, Brot und Wein und Sternenhimmel".

Es ist von daher anzunehmen, daß die Tatsache, daß Wolfskehl sich in Neuseeland aufhielt, mit zu Singers Entscheidung beigetragen hat, sich - als er Japan verlassen mußte - im gleichen Teil der Erde niederzulassen. Singer war bei seiner Abreise ungefähr acht Jahre in Japan gewesen. Er hatte bei seiner Ankunft - von Lafcadio Hearns Schilderungen geprägt - ein romantisches, exotisches, stimmiges, traditionelles, "ganzes" Japan erwartet. Statt dessen fand er dort Menschen, deren Augen und Haltung ,schlaff und verzerrt“ waren „wie die führerlosen Seelen, die aus allem, was sie erfahren, nur Antriebe zu ärgerer Aufdröselung entnehmen. Man hatte damals die Parole Erogoro-nonsense ausgegeben, was erotischgrotesk-sinnlos heißen sollte und für die private Lebensführung auszureichen schien, während sich bei den leidenschaftlichsten Jüngeren der Drang nach Tat in endlosen Diskussionen und vielgespaltenen Konspirationen in kommunistischen Geheimzirkeln zu

38 Ebd., S. 1287.

39 Ebd., S. 1281.

40 Singer, "Bericht", S. 598 f. 
entladen suchte, nicht selten in Hörigkeit gegen Frauen vom Schlage der frühen russischen Revolutionärinnen, und offenbar vom fernen Moskau gelenkt. Die älteren Männer erschienen hilflos, skeptisch und entschlußunfähig. Die Parteien verloren von Jahr zu Jahr mehr an Kredit. Ein Aufstand, der durch Preissturz und Pachtlasten zerdrückten Bauern schien von Tag zu Tag wahrscheinlicher". ${ }^{41}$ Er sah ein Land, geprägt von überstürzter Ausdehnung und Industrialisierung, von Nationalismus, von politischem Ungleichgewicht hinter einer Fassade westlicher Zivilisation, hinter der er erst nach einiger Zeit traditionelle japanische Elemente entdeckte. Singer blieb auch nach mehr als acht Jahren in Japan ein Fremder. Ôta berichtet, ${ }^{42} \mathrm{da} ß$ er während seiner Tokyoter Zeit abgeschieden in Kamakura lebte. An den üblichen gemeinsamen Freizeitvergnügen mit seinen Kollegen nahm er lange Zeit gar nicht, später nur sporadisch teil. Auch war er - im Gegensatz zur Ansicht Pawlowskys ${ }^{43}$ und Wilhelms, ${ }^{44}$ die dazu neigen, ihn in dieser Hinsicht etwas zu mystifizieren - wie bemerkt, wenig populär bei seinen Schülern und Studenten. Einigen wenigen, die ihm intellektuell gewachsen waren, war er ein hervorragender Lehrer. Den meisten jedoch erschien er abschreckend, geheimnisvoll, ernst und unverständlich. ${ }^{45}$ Singer war während seiner japanischen Zeit immer auf der Suche nach den traditionellen Elementen in der japanischen Kultur, besonders in der.Dichtung und machte sich intensiv mit Kunstformen wie der des Ikebana, der Teezeremonie und der Lyrik vertraut und erreichte hier wohl einen erstaunlichen Kenntnisstand. 46

Ôta erzählt, 47 daß ein Kollege ihm im Januar 1932 eine Einladung zum Go-uta-kai-hajime, einer traditionellen Gedichtlesung am kaiserlichen Hof zu Neujahr, verschaffte, bei der er mit erstaunlicher Sachkenntnis die vorgetragenen Gedichte kommentierte. Bei Eschbach 48 dient dies u.a. als Beweis für seine wohl hervorragenden Japanischkenntnisse. Anwesende bei dieser Gedichtlesung berichten jedoch, daß die Gedichte für ihn übersetzt wurden. ${ }^{49}$ Er selbst er-

41 Ebd., S. 592.

42 Ôta, "Kurto Jinga", in: Tôkyo University Press 144 (1984), S. 21.

43 Pawlowsky, "Vorwort", S. XI.

44 Wilhelm, "Vorwort", S. $13 \mathrm{f}$.

45 Ôta, Hidemichi, "Ôsutoraria no Jingâ (Singer in Australien)", in: Tôkyo

University Press 1962, S. 28.

46 Ôta, "Kurto Jinga", in: Tôkyo University Press 145 (1984), S. 22.

47 Ebd., S. 23.

48 Eschbach, "Kurt Singer", S. 3 f. 
wähnt zudem, daß besonders in den ersten Jahren, die „Schwierigkeiten sprachlicher Verständnis“ seinen Gesprächen in Japan „die engsten Schranken“ zogen.50 Auch dies ist wohl ein Grund für sein Fremdbleiben in Japan.

Singer verläßt im August 1939 Japan mit dem Ziel Australien. Nach seiner Internierung entsteht hier zuerst die große Studie The Idea of Conflict. Da diese direkt zu Wolfskehl keinen Bezug hat, sei sie an dieser Stelle nur der Vollständigkeit halber erwähnt.

Überblickt man die Korrespondenz zwischen Singer und Wolfskehl aus dieser Zeit, 51 so findet man Diskussionen über Abhandlungen Singers zum Judentum, wie z. B. die Kain-Exegese, über allgemeine weltpolitische Themen und oft wird der Wunsch beider Schreiber deutlich, sich doch einmal zu treffen, wozu es nicht gekommen ist. 52 Auch weitete sich das Verhältnis zwischen Singer und Wolfskehl im Grunde zu einem Dreieck aus über die Person von Hans Brasch. Er gehörte ebenfalls in den George-Umkreis und war - von Hause aus Ingenieur - poetisch interessiert und tätig. Brasch und Singer standen in persönlichem Kontakt in Melbourne, während Wolfskehl mit Brasch in brieflichem Kontakt stand. Karl Wolfskehl rekurriert in Briefen aus dieser Zeit auf Singer und Brasch als „Sie beide". 53

Als es Singer schließlich gelang, eine Anstellung in Sydney zu bekommen, verließ er schweren Herzens Brasch in Melbourne. Trotz seiner Anstellung blieben seine Lebensumstände äußerst kärglich, das Verhältnis zu seinen Studenten war ähnlich wie in Japan, er war frustriert vom australischen Universitätssystem und er war einsam trotz einer - wie auch immer gearteten - Beziehung zu einer Kollegin. ${ }^{54}$ Er flüchtete sich wohl auch deshalb in Produktivität.

Neben anderem arbeitete Singer an einem seiner Hauptwerke über Japan, der Karl Wolfskehl gewidmeten Studie Mirror, Sword, and Jewel. Diese Widmung ist wohl damit zu erklären, daß Singer gerade Wolfskehl immer wieder kleinere Abhandlungen zu dieser Thematik geschickt hatte und dort auf großes Interesse gestoßen war.

Mirror, Sword, and Jewel wurde erst elf Jahre nach seinem Tod

49 Ôta, "Kurto Jinga", in: Tôkyo University Press 144 (1984), S. 24.

50 Singer, "Bericht", S. 597.

51 Vgl. BaN II, S. 325-336.

52 Ebd., S. 332.

53 Ebd., S. 329 und 1097.

54 Ôta, Hidemichi, "Ôsutoraria no Jingâ (Singer in Australien)", in: Tôkyo University Press 1962, S. 28. 
1973 von Richard Storry bei dem Londoner Verlag Croom Helm herausgegeben. Singer schlo $\beta$ das vollständige englischsprachige Manuskript 1950/51 in Sydney ab. Daneben existieren Bruchstücke und Entwürfe zu diesem Buch, die zum Teil wohl noch aus seiner japanischen Zeit stammen. Interessant ist, daß das Manuskript nach der englischen Veröffentlichung 1991 unter Einbeziehung der ursprünglichen deutschen Dokumente wieder ins Deutsche rückübertragen wurde unter dem Titel Spiegel, Schwert und Edelstein. Strukturen des japanischen Lebens. 55

Dieses Buch über Japan ist in drei Komplexe eingeteilt, die Singer mit "Charakteristika“, „Strukturen“ und „Werte“ überschreibt. Deutlich zum Ausdruck kommt Singers geistige Nähe zu George in der Betonung des Heroischen, der Heraushebung antiker Tugenden und der ,hohen Einschätzung des Genies als Wirkungsfaktor in der Geschichte". .56

Singers Grundhaltung in diesem Buch ist dadurch gekennzeichnet, daß er kein grundsätzliches Primat des Westens gegenüber Japan ansetzt. Im Gegenteil, er konstatiert, daß man in vielen Aspekten von Japan lernen kann, zum Beispiel: „In gleißendem Licht entsteht kein Leben. Diese Lehren [die in Japan herrrschen] haben die fortgeschrittensten Nationen des Westens noch immer zu lernen oder wiederzulernen". 57 Diese Konsequenz resultiert sicherlich einerseits aus Singers Faszination von diesem Land, andererseits aber auch aus der Tatsache, daß dieses Buch als ein Appell gegen die deutschen Entwicklungen gedeutet werden kann.

Singer beeindruckt durch eine Vielzahl an Detailkenntnissen und die Breite der behandelten Aspekte: Geschichte, Tradition, Rituale, Gesellschaft, Religion, Nation, allgemeine soziale Fragestellungen (Verstädterung), Politik, Mythen etc. Auch sein Einblick in Differenzierungen, besonders im soziologischen Bereich (InnenAußen, uchi-soto) 58 ist bemerkenswert. Singer behauptet nicht, Japaner seien höflich, sondern konstatiert, japanische Höflichkeit variiere - je nach Zugehörigkeit des Interaktionspartners zur eigenen Gruppe oder einer fremden Gruppe. Diese Differenzierung nach Interaktionsraum - ist eine sehr zentrale Erkenntnis.

Schon bei seinem Konzept von japanischer Individualität jedoch scheinen Widersprüche auf. Seine Aussage: „Überhaupt ist es ein Irrtum anzunehmen, daß die Japaner von Natur aus weniger selbst-

$55 \mathrm{Vgl}$. Anm. 1.

56 Wilhelm, "Vorwort", S. 22.

57 Singer, Spiegel Schwert und Edelstein, S. 124.

58 Ebd., S. 187. 
süchtig, ich-betont und eigenwillig seien als Westländer"59 läßt aufhorchen und zustimmen, stellt sie doch der sonst so gern propagierten "Gruppenmentalität" ein wirklichkeitsnäheres Konzept entgegen. Singer schränkt dies allerdings gleich wieder ein, wenn er unter der Überschrift „Persönlichkeit und Ich"60 bemerkt: „Es gibt in Japan, dem alten wie dem neuzeitlichen, Männer und Frauen, die wir als Persönlichkeiten bezeichnen dürfen, Gestalter ihres eigenen Charakters und eines eigenen Lebenstils [...]. Doch finden sich solche Fälle fast immer in den Grenzbezirken der japanischen Gesellschaft: bei den großen Mönchen, die der Welt entsagt haben, und bei den großen Kurtisanen (jorô) und Sängerinnen (geisha), die außerhalb der bürgerlichen Bannmeile leben [...].61 Es stellt sich dadurch die Frage, ob Individualität in Japan sich für ihn dann doch als Ausnahmephänomen darstellt.

Problematisch ist ebenfalls die monolithische Sehweise von Japanern und Westländern, die Singer durchgängig einander gegenüberstellt. Singer spricht an einer Stelle vom „typischen“ Japaner, den er sich mit geschlossenen Augen vorstellt, ${ }^{62}$ hier gibt es keine Differenzierung nach Geschlecht, historischer Situation oder sozialer bzw. regionaler Herkunft etc. Verfolgt man diesen Gedanken weiter und versucht, sich den „typischen Westländer" mit geschlossenen Augen vorzustellen, ist man durchaus mit einigen Schwierigkeiten konfrontiert.

Auch hebt Singer in seinen Betrachtungen immer wieder auf Stereotype über Japaner ab:

Japaner sind passiv63 und autoritätsabhängig, ${ }^{64}$ die japanische Sprache ist vage, ausweichend, andeutend etc., ${ }^{65}$ Japaner sind generell harmonisch und konfliktscheu,66 etc. Hier handelt es sich um Argumente, die die exotische Eigentümlichkeit und die „Unnachahmbarkeit" Japans ${ }^{67}$ unterstreichen. Weitere interessante Beobachtungen Singers sind z. B. diejenige, Japan habe den „Bereich des Genius auf ein bloßes Mindestmaß reduziert [...]. Es hat wenige schöpferische Persönlichkeiten hervorgebracht und den wenigen

\footnotetext{
59 Ebd.

60 Ebd., S. 129.

61 Ebd., S. 131.

62 Ebd., S. 255.

63 Ebd., S. 67.

64 Ebd., S. 189.

65 Ebd., S. 123.

66 Ebd., S. 150.

67 Ebd., S. 255.
} 
eine geringe Schaffensbreite eingeräumt".68

Außerdem postuliert Singer die Prägung der japanischen Nation durch ihre natürliche Umwelt, eine Sicht, die zwar sicherlich zeitgebunden verständlich, bei näherem Hinsehen aber dennoch problematisch und überholt ist. ${ }^{69}$

Dieses sind Aspekte, die es gerechtfertigt sein lassen, diesem Buch als Zeitdokument durchaus seinen Platz einzuräumen. Rezensenten wie Donald Richie allerdings haben diesem Buch einen anderen Weg gewiesen. Am 3. November 1979 erschien Richies Sammelrezension von Büchern über Japan in Japan Times Weekly. Er bespricht hier fünf Standardwerke über Japan (Basil Hall Chamberlain Things Japanese, Lafcadio Hearn Japan: An Attempt at Interpretation, Bernhard Rudofsky The Kimono Mind und Roland Barthes L'Empire des Signes) und stellt Singers Werk unter diesen als das bei weitem Herausragende dar. ${ }^{70}$ Er geht sogar so weit, es als das beste Buch über Japan, das er je gelesen habe, zu bezeichnen. Eine ausführliche Begründung für diese Wertung fehlt allerdings. ${ }^{71}$ Derartige Rezensionen - wie auch die von Frank Tuchy im Encounter Magazine im Mai 1973 - messen dem Buch eine Bedeutung zu, die es - ungeachtet der oben angeführten problematischen Aspekte bis heute offensichtlich beibehält. So stimmt Achim Eschbach 72 in jüngster Zeit aus methodischen Gründen der Einschätzung Richies uneingeschränkt zu.

An dieser Stelle schließt sich die Frage an, warum man Singers Überlegungen nicht einfach als Zeitdokumente stehen lassen und sich diesem Strom der schrankenlos anerkennenden Rezeption anschließen kann. Dafür gibt es eine einfache Erklärung. Singers Buch gehört zur sogenannten Nihonjinron-Literatur. Dabei handelt es sich um Betrachtungen der japanischen Nation als Ganzheit. Im Vordergrund stehen die Herausarbeitung eines Nationalcharakters und typischer Eigenschaften, wie Homogenität, Gruppismus, Harmonie und Konsens. Wie man bei Singer sieht, ist das Bild der japanischen Nation überaus positiv. Was dahinter steht, ist die Tatsache, daß man in Japan einen Spiegel dessen sucht, was man sich selber wünscht. Dies ist gerade in politisch schwierigen Situationen wie denjenigen, in denen sich Singer bei der Entstehung des Buches befand, der Fall. Damit schafft man allerdings Vorurteile, Grenzen

68 Ebd., S. 240.

69 Ebd., S. $64 \mathrm{ff}$.

70 Vgl. dazu Ôta, "Kurto Jinga”, in: Tôkyo University Press 144 (1984), S. 26 f.

71 Ôta, "Kurto Jinga", in: Tôkyo University Press 145 (1984), S. 27.

72 Eschbach, "Kurt Singer", S. 9. 
und Trennungslinien, die Japan nicht aus der Ecke der Exotik, des Andersseins und der mystifizierten Unverständlichkeit herauskommen lassen. Zumal dieses Image von den Japanern selbst, wo es probat erscheint, gerne aufgegriffen wird. Will man es Japan ermöglichen, einen gleichberechtigten Platz in der internationalen Gemeinschaft auf allen Ebenen einzunehmen, muß man der Mystifizierung Japans, wie sie in Singers Buch aufscheint, kritisch entgegentreten.

Mirror, Sword, and Jewel stellt sicherlich ein wertvolles Zeitdokument dar, wohl aber nicht das beste Buch über Japan, das je geschrieben worden ist. $\mathrm{Zu}$ verstehen ist es, wie auch Singers andere Abhandlungen zu Japan, aus seiner Suche nach dem Menschen als "Ganzem", einer Suche, auf der sich Wolfskehl ebenso wie er befindet. Eine Suche, die zwei geistig verwandte, durch das gemeinsame Schicksal der Emigration verbundene, daher mit ähnlichen Problemen konfrontierte Persönlichkeiten verbindet. 